\title{
Soft QCD Measurements in the Forward Direction with the LHCb Experiment
}

\author{
Sebastian $\bar{S}$ chleich on behalf of the LHCb Collaboration
}

The 19th Particles and Nuclei International Conference (PANIC11)

MIT Cambridge MA

28.07.2011 


\section{Outline}

1. The LHCb experiment

\section{PID system}

LHCb data

2. $K_{S}^{0}$ production

3. V0 ratios

$\bar{\Lambda} / \Lambda$ ratio

$\bar{\Lambda} / K_{S}^{0}$ ratio

4. $\bar{p} / p$ ratio

5. $\phi$ production

6. Charged track multiplicities 


\section{The LHCb experiment}
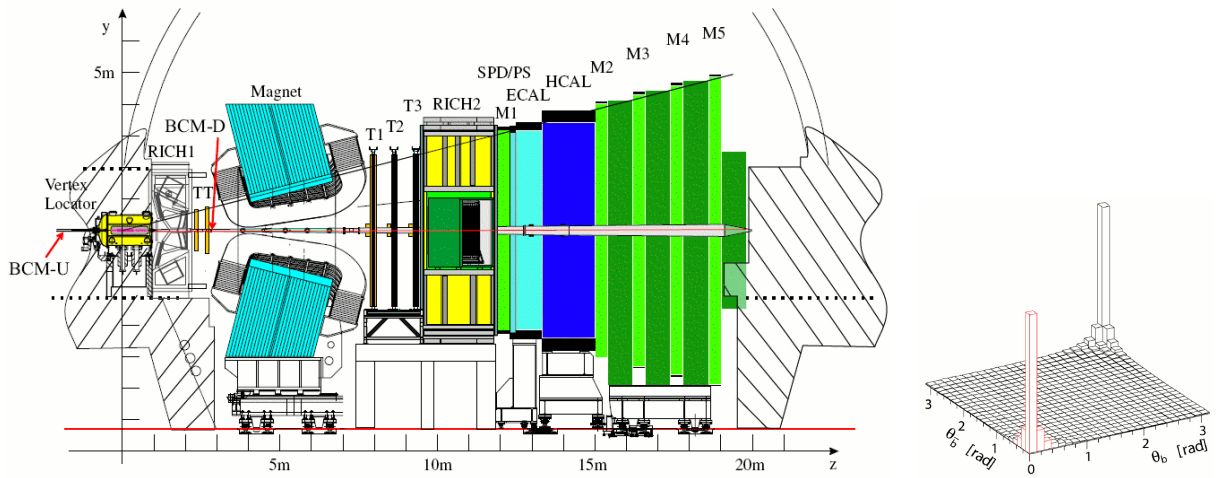

- aims at precision measurements of $C P$ violation in the $b$ sector

- single arm forward spectrometer $1.9<\eta<4.9$ (backwards: VELO "Pileup Unit")

- excellent lifetime resolution: VELO, $5 \mathrm{~mm}$ distance to beam, movable 


\section{PID system}

dedicated PID system based on RICH detectors:

- RICH1:

- Aerogel $p<10 \mathrm{GeV} / c$

- $\mathrm{C}_{4} \mathrm{~F}_{10} \quad p<60 \mathrm{GeV} / c$

- $\mathrm{RICH} 2$ :

$$
\text { - } \mathrm{CF}_{4} \quad 15<p<100 \mathrm{GeV} / c
$$

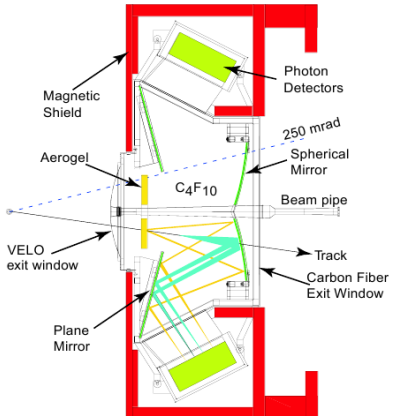

important ingredient e.g. to $\bar{p} / p$ and $\phi$ studies
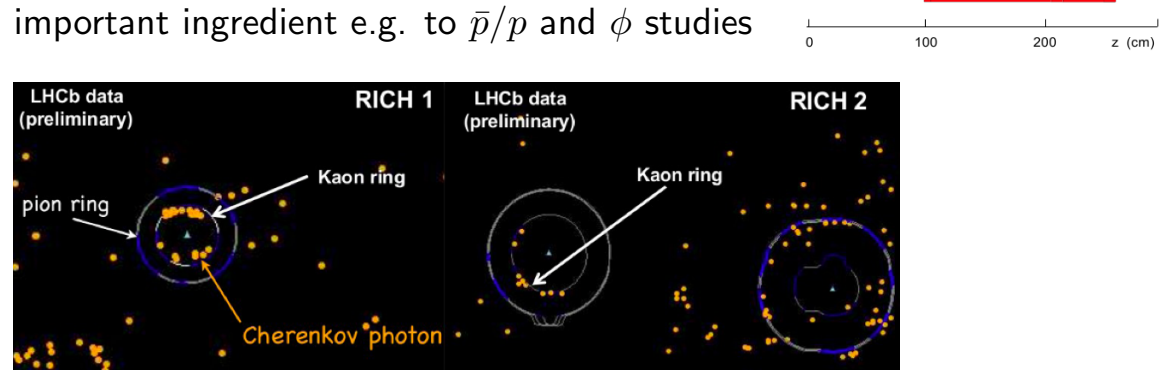


\section{Recorded luminosity}
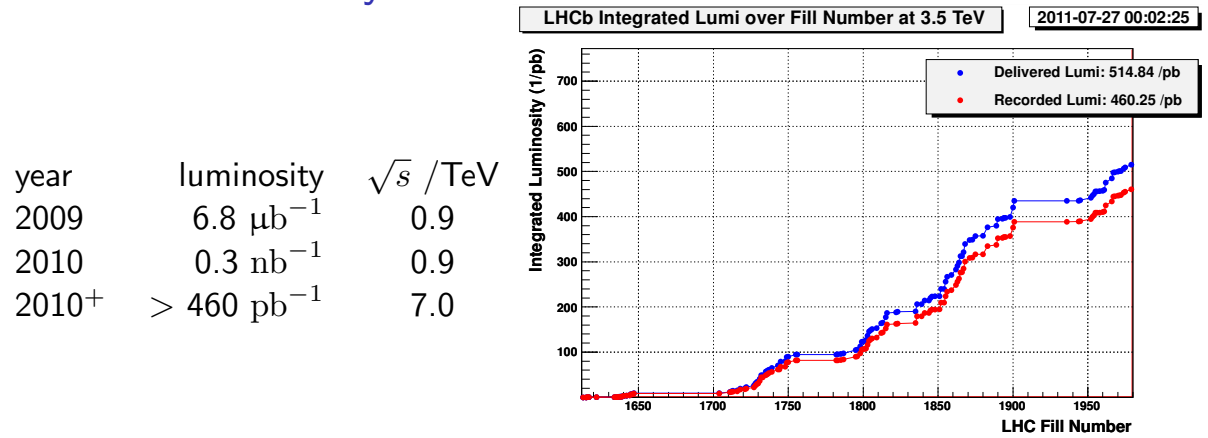

...90\% data taking efficiency!

in this talk:

\begin{tabular}{l|cc} 
& $\sqrt{s}=900 \mathrm{GeV}$ & $\sqrt{s}=7 \mathrm{TeV}$ \\
\hline$K_{S}^{0}$ cross-section & $\mathrm{X}$ & $\mathrm{X}$ \\
$\phi$ cross-section & & $\mathrm{X}$ \\
$\mathrm{V} 0$ ratios & $\mathrm{X}$ & $\mathrm{X}$ \\
$\bar{p} / p$ ratio & $\mathrm{X}$ & $\mathrm{X}$
\end{tabular}




\section{$K_{S}^{0}$ production}

\section{Physics Letters B 693 (2010) pp. 69-80 arXiv:1008.3105v2}

- $K_{S}^{0}$ reconstructed in $K_{S}^{0} \rightarrow \pi^{+} \pi^{-}$

- analyzed data from pilot run 2009

- low requirements on reconstruction, no PID...

- ideal 'first physics' channel

- testing ground for detector understanding/calibration

- $K_{S}^{0}$ decay far away from primary vertex

- two separate analyses done (with/without VELO information)

key systematics:

luminosity

tracking efficiency
$12 \%$

$10 \%$ 


\section{$K_{S}^{0} p_{T}$ spectra at $\sqrt{s}=900 \mathrm{GeV}$}
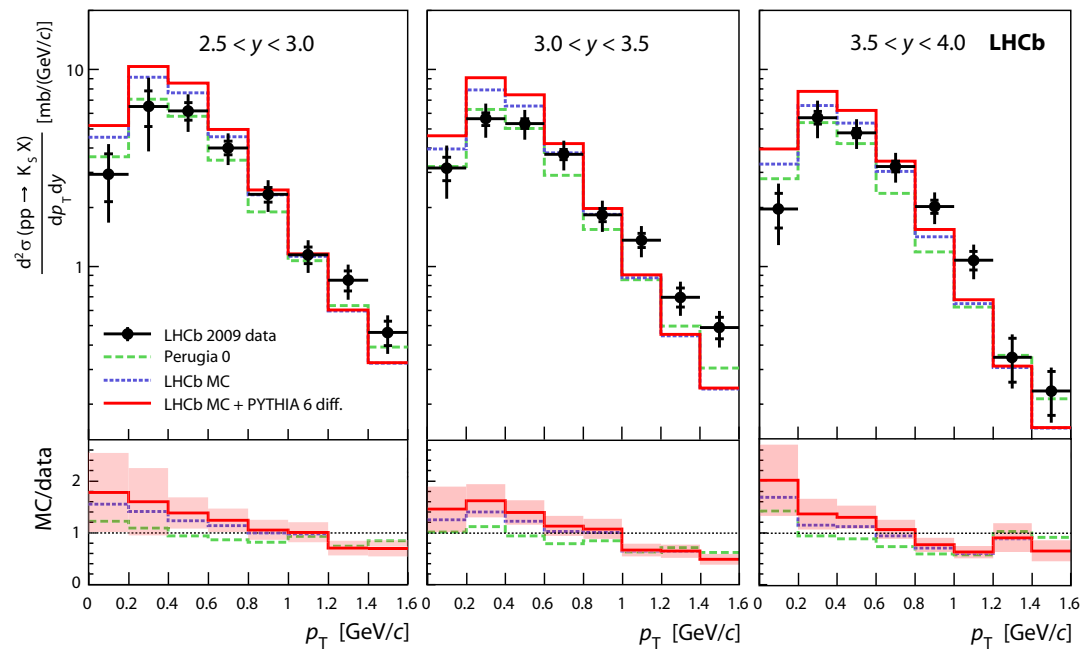

- harder $p_{T}$ spectrum in data than MC 


\section{Comparison with other experiments}

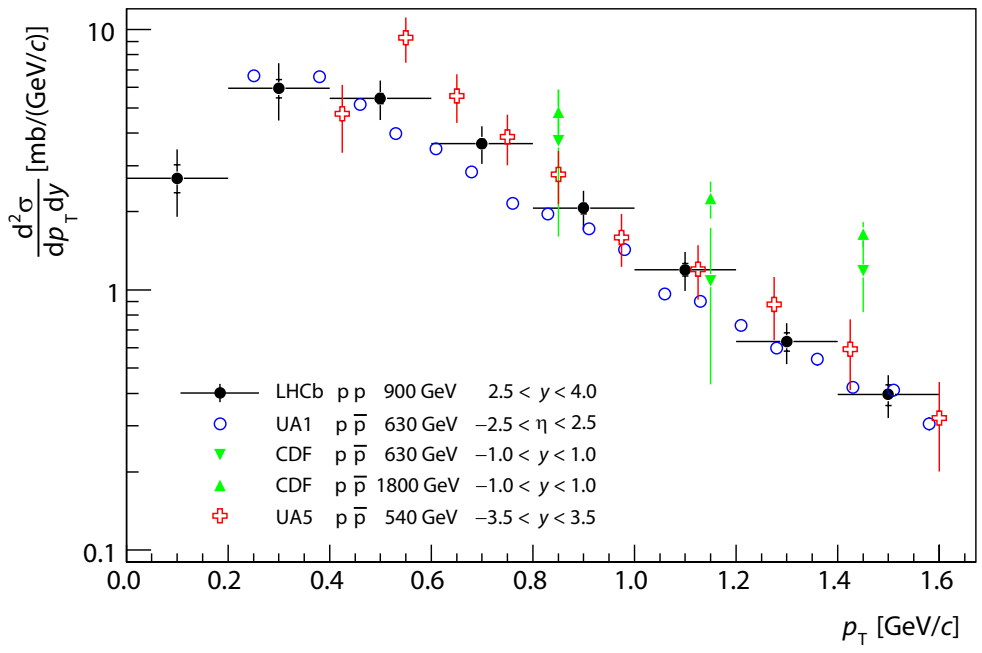

- first measurement at $\sqrt{s}=900 \mathrm{GeV}$

- $y$ and $p_{T}$ range extended 


\section{V0 ratios}

\section{arXiv:1107.0882v2 (submitted to JHEP)}

identification:

$$
\begin{array}{ll}
\Lambda & \Lambda \rightarrow p \pi^{-} \\
\bar{\Lambda} & \bar{\Lambda} \rightarrow \bar{p} \pi^{+} \\
K_{S}^{0} & K_{S}^{0} \rightarrow \pi^{+} \pi^{-}
\end{array}
$$

- $K_{S}^{0}$ and $\Lambda$ selection based on impact parameters

- independent from luminosity

- low systematic uncertainties (cancel partially) 


\section{$\bar{\Lambda} / \Lambda$ ratio}
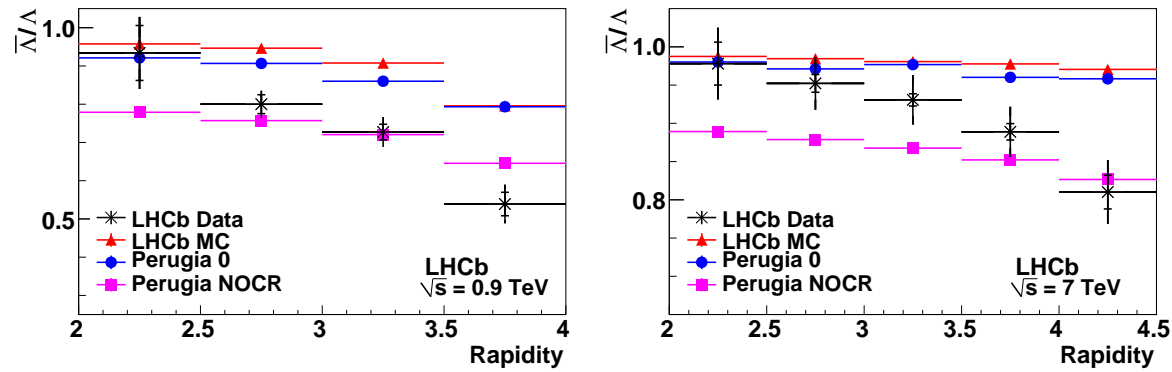

$\Rightarrow$ rapidity dependence:

- at low rapidity, ratio like Perugia 0

- at high rapidity, more like Perugia NOCR 


\section{$\bar{\Lambda} / \Lambda$ ratio $-p_{T}$ dependence}
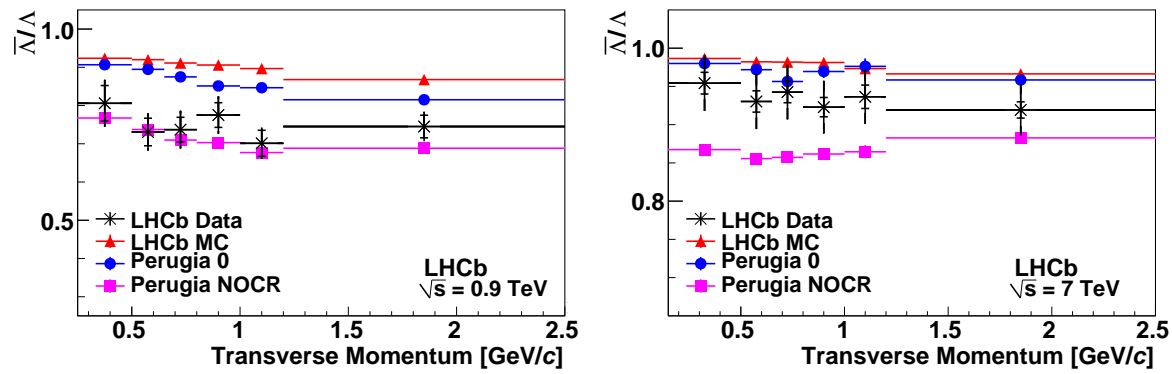

$\Rightarrow$ energy dependence:

- at $\sqrt{s}=900 \mathrm{GeV}$, Perugia NOCR fits better

- at $\sqrt{s}=7 \mathrm{TeV}$, data closer to Perugia 0 


\section{Baryon transport $\bar{\Lambda} / \Lambda$}

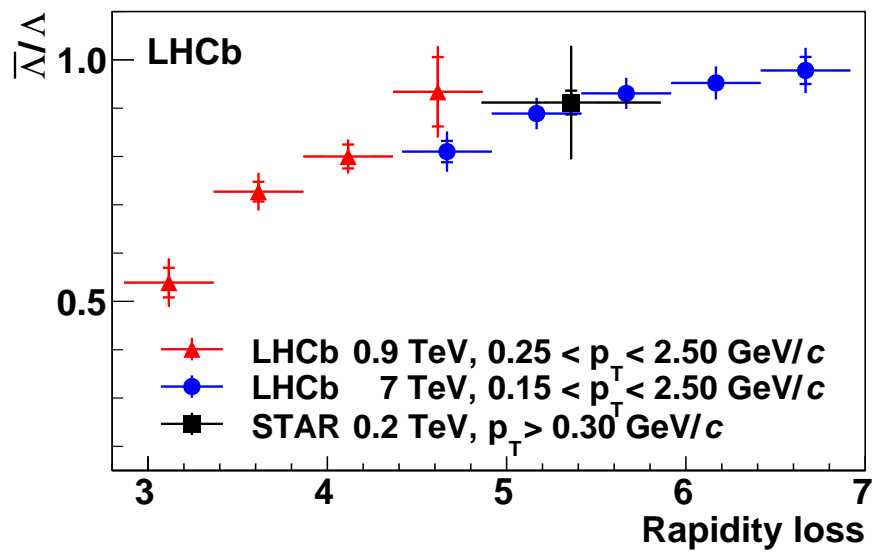

Rapidity loss $\Delta y=y_{\text {beam }}-y_{\text {baryon }} \quad y_{\text {beam }} \approx \ln \frac{2 E}{m}$

- consistency between $\sqrt{s}=900 \mathrm{GeV}, 7 \mathrm{TeV}$ and previous measurement 


\section{$\bar{\Lambda} / K_{S}^{0}$ ratio}
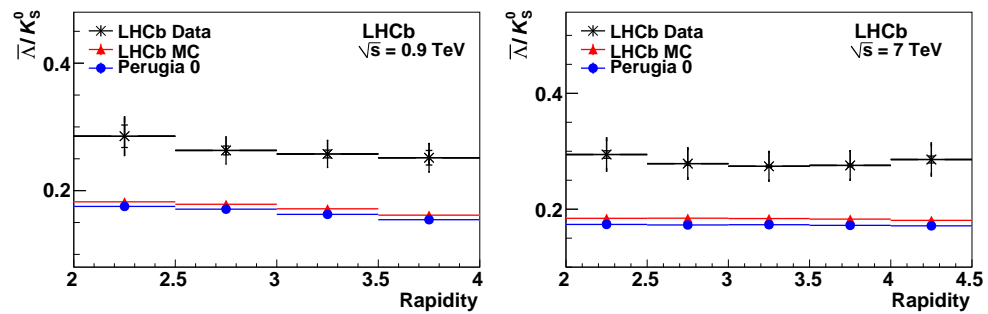

- ratio underestimated by $\mathrm{MC}$ at both beam energies
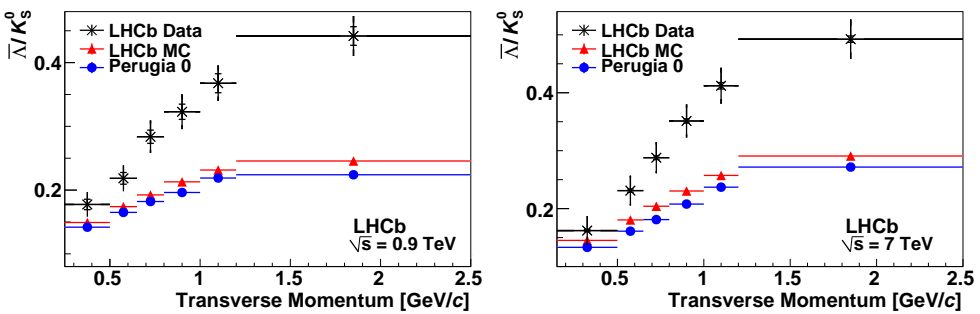

- the higher $p_{T}$, the further off is the $\mathrm{MC}$ 


\section{$\bar{\Lambda} / K_{S}^{0}$}

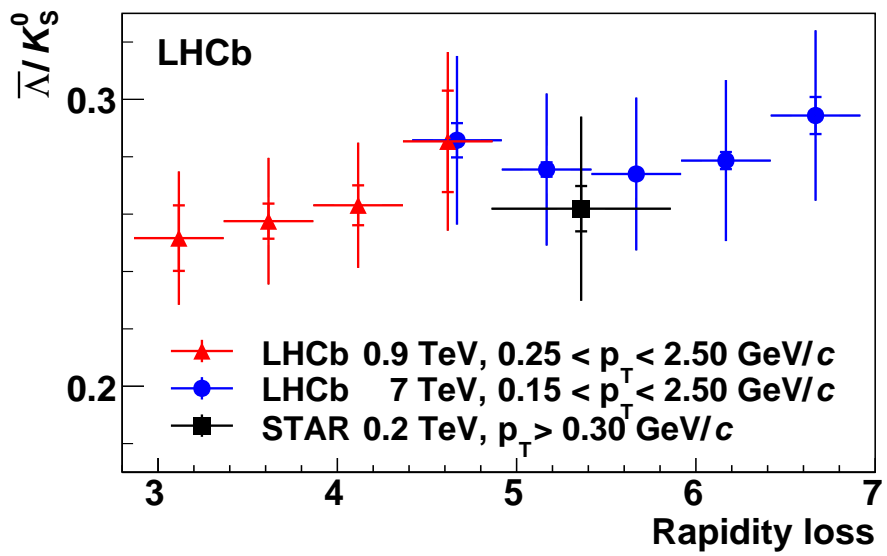

- consistency between $\sqrt{s}=900 \mathrm{GeV}, 7 \mathrm{TeV}$ and previous measurement 


\section{$\bar{p} / p$ ratio $-\mathrm{PID}$}

- analysis strongly depends on PID system

- PID calibrated on data:

- $\pi$ and $\mathrm{p}$ from $K_{S}^{0} \rightarrow \pi^{+} \pi^{-}$and $\Lambda \rightarrow p \pi$

- $\mathrm{K}$ from $\phi \rightarrow K K$
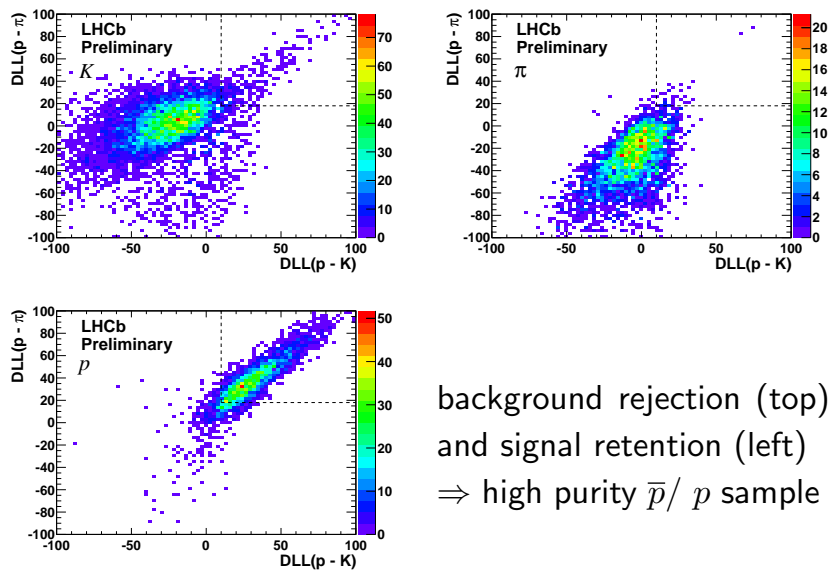

background rejection (top) and signal retention (left) $\Rightarrow$ high purity $\bar{p} / p$ sample 
$\bar{p} / p$ ratio

\section{preliminary: CERN-LHCb-CONF-2010-009}
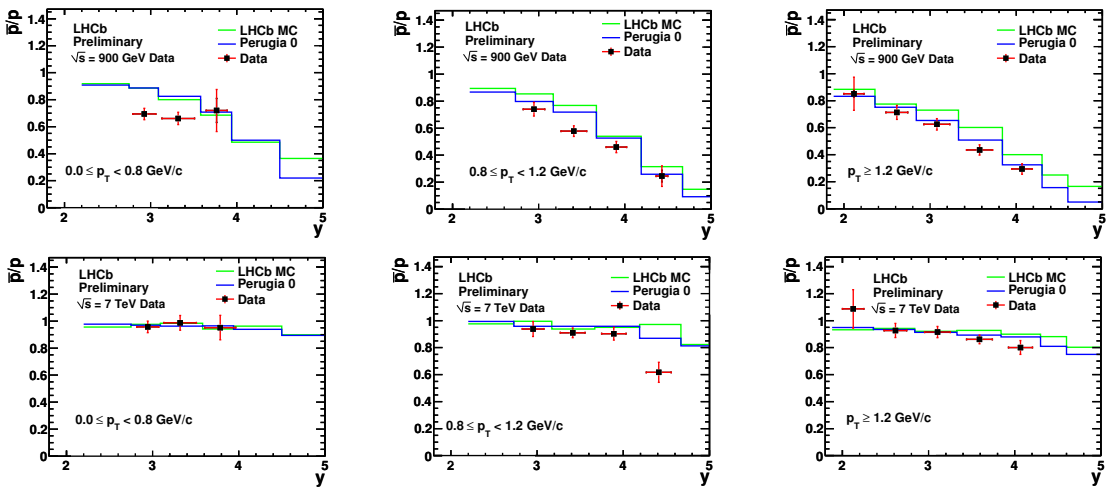

- ratio overestimated at $\sqrt{s}=900 \mathrm{GeV}$

- good MC-data agreement at $\sqrt{s}=7 \mathrm{TeV}$ 


\section{Baryon transport $\bar{p} / p$}

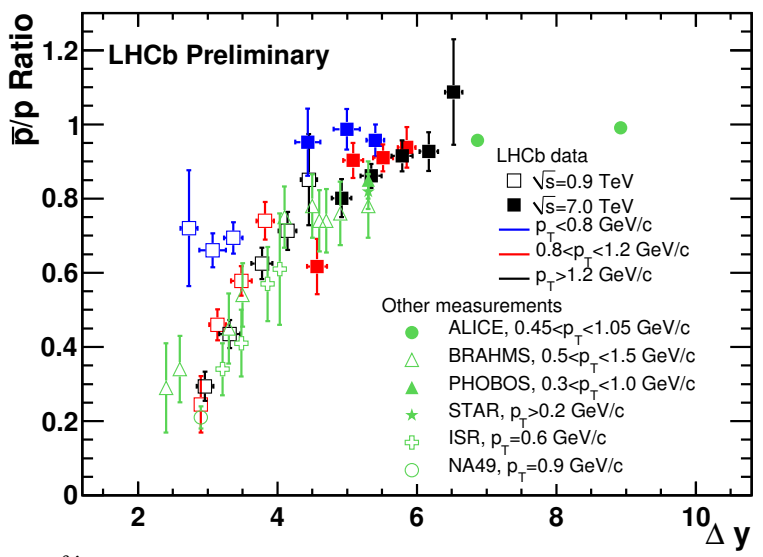

$\Delta y=y_{\text {beam }}-y_{\text {baryon }}$

- consistency with other measurements, in particular at high $p_{T}$ 


\section{$\phi$ production}

\section{arXiv:1107.3935 (submitted to PLB)}

- $\phi$ production cross-section measured in bins of transverse momentum $p_{T}$ and rapidity $y$

only reconstruction efficiency relies on $\mathrm{MC}$

RICH PID cut efficiency determined on data (tag\&probe)

- apply PID cut on at least one kaon

- subsequently cut on the second kaon

- deduce PID cut efficiency

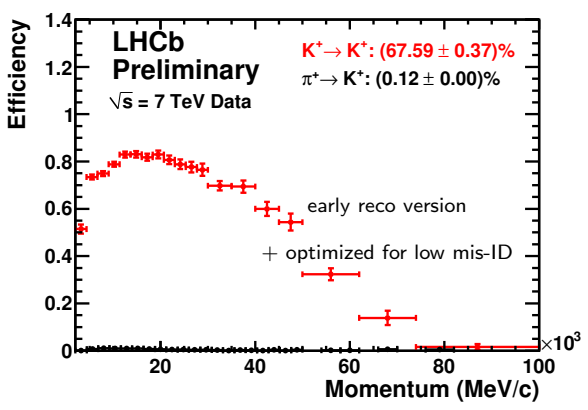




\section{$\phi$ production - tag-and-probe}

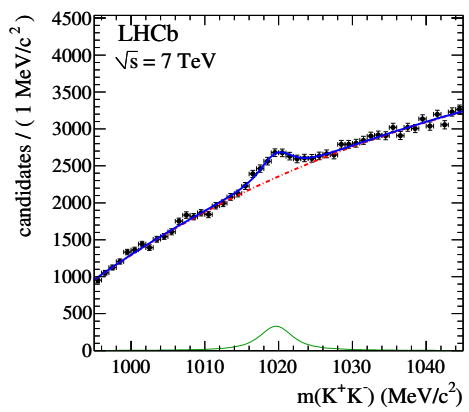

tag sample

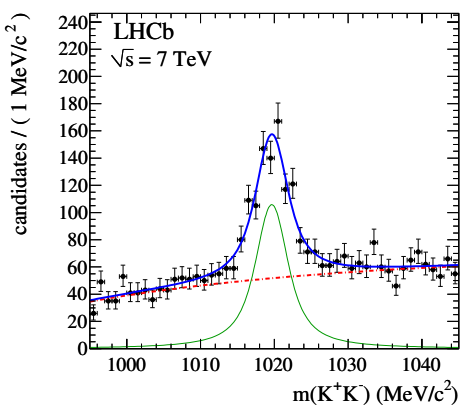

probe sample

\section{$\Rightarrow$ PID efficiency}




\section{$\phi$ cross-section projections}
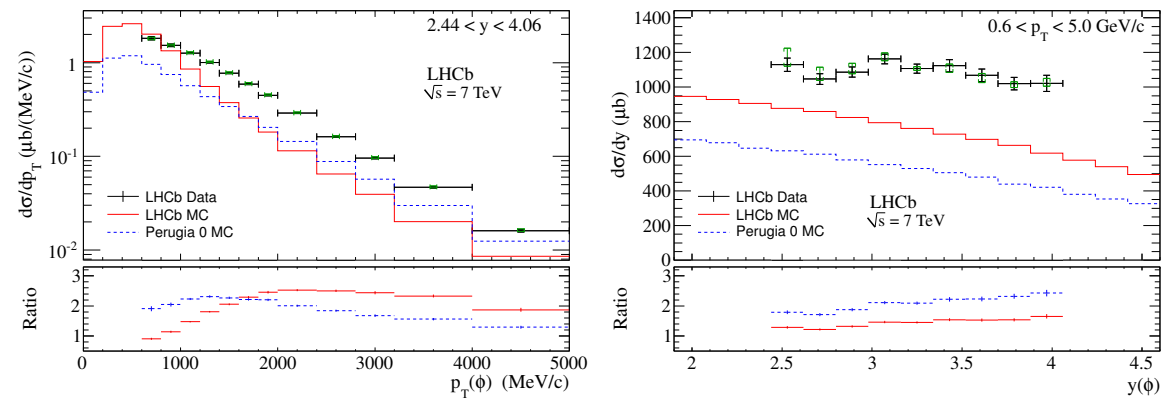

- $\phi$ production underestimated in the measured kinematic range by both, LHCb-MC and Perugia 0

- $p_{T}$ spectrum different from $\mathrm{MC}$ 


\section{Charged track multiplicities}

- particles counted by reconstructing tracks in the VELO outside magnetic field, no momentum measurement

- only tracks from luminous region, non-prompt contamination (5-10\%) corrected using MC

- no explicit momentum cut, efficiency drop versus low $p_{T}$ corrected for

- pile-up is corrected for

- detector resolution is unfolded 


\section{Charged track multiplicities}

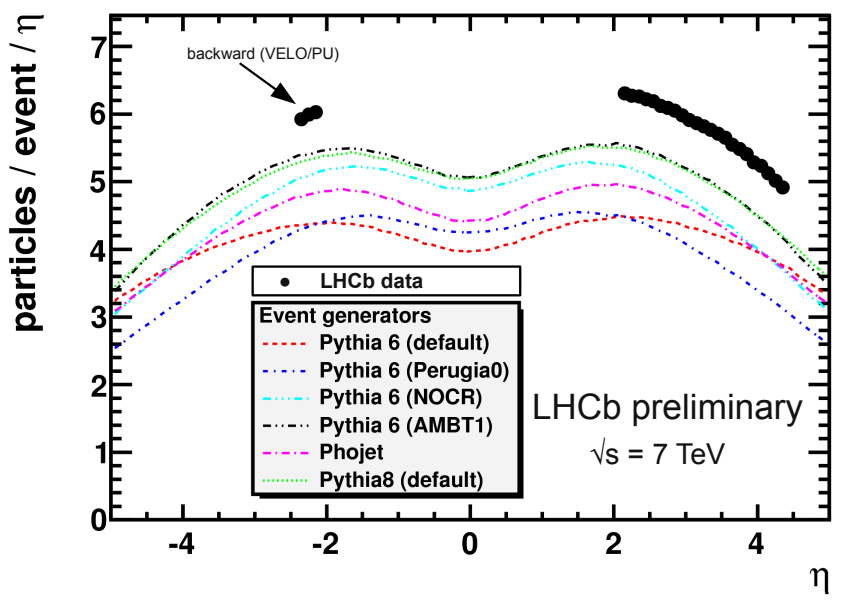

normalized to events with at least one charged particle in the forward acceptance $2.0<\eta<4.5$ 


\section{Charged track multiplicities}

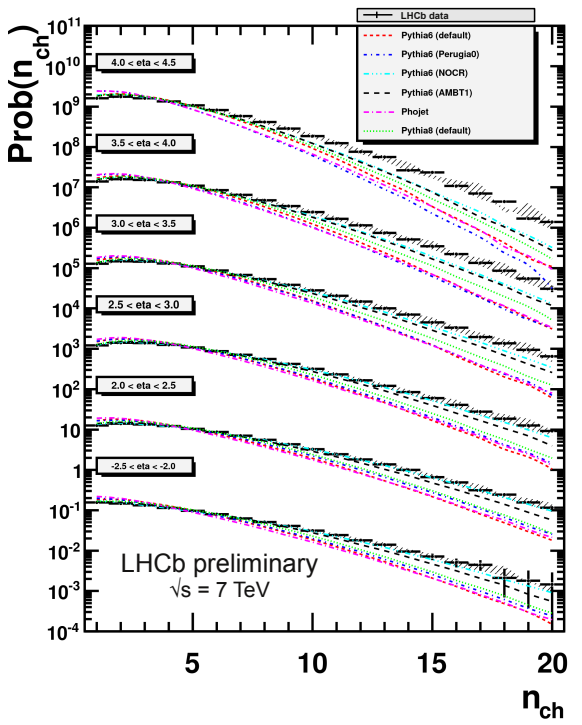

Hard interactions: normalized to events with at least one charged particle with $p_{T}>1 \mathrm{GeV} / c$ in the forward acceptance $2.0<\eta<4.5$

best agreement of data with Pythia 6 (NOCR) 


\section{Conclusions}

LHCb delivers splendid input to Soft QCD physics in the high- $\eta$ region

- $K_{S}^{0}$ production at $\sqrt{s}=900 \mathrm{GeV}$ harder $p_{T}$ spectrum as compared to $\mathrm{MC}$

- $\bar{\Lambda} / \Lambda$ ratio lower than Perugia 0 , in particular at high $y$

- $\bar{\Lambda} / K_{S}^{0}$ ratio higher than Perugia 0

- $\bar{p} / p$ ratio slightly lower than Perugia 0 at $\sqrt{s}=900 \mathrm{GeV}$

- $\phi$ production at $\sqrt{s}=7 \mathrm{TeV}$ above Perugia 0 in the considered kinematical range

- track multiplicity generally higher on data than on MC - better agreement for hard events only 